\title{
Gestão e classificação dos estoques em hospitais da rede privada de médio porte no
}

\author{
Brasil \\ Inventory management and classification in private medium-sized hospitals in Brazil \\ Manejo y clasificación de inventario em hospitales privados medianos en Brasil
}

Recebido: 26/09/2021 | Revisado: 02/10/2021 | Aceito: 04/10/2021 | Publicado: 05/10/2021

\author{
Cibele Del Hoyo Pacheco \\ ORCID: https://orcid.org/0000-0001-6177-0007 \\ Universidade Federal de São Paulo, Brasil \\ E-mail: cibeledhp@gmail.com \\ Maykon Anderson Pires de Novais \\ ORCID: https://orcid.org/0000-0001-8069-4927 \\ Universidade Federal de São Paulo, Brasil \\ E-mail: amaykon@gmail.com \\ Márcia Mello Costa De Liberal \\ ORCID: https://orcid.org/0000-0002-2589-1802 \\ Universidade Federal de São Paulo, Brasil \\ E-mail: deliberal@uol.com.br
}

\begin{abstract}
Resumo
Introdução: A gestão racional dos estoques consiste em uma ferramenta indispensável para que uma unidade hospitalar possa ser gerida de maneira competitiva, em especial no caso das redes privadas que estão sujeitas à pressão do mercado e das seguradoras de saúde. Objetivos: Analisar o consumo de suprimentos hospitalares com base em classificações que auxiliam os processos estratégicos na gestão hospitalar. $\mathrm{O}$ estudo justifica-se pela carência de parametrização e input de dados relacionados aos itens do suprimento de hospitais geridos por um sistema hospitalar de saúde no Brasil em termos de informações centralizadas para as tomadas de decisão dos gestores hospitalares. Método: O estudo é de natureza observacional exploratória e buscou subsídios necessários para aprimorar a relação entre Indicadores básicos x Hospital na gestão dos suprimentos hospitalares. Foi priorizada a percepção dos gestores da saúde e como esses dados pesquisados podem ser aplicados de maneira estruturada em decisões gerenciais. A amostra teve como foco seis hospitais gerais privados de saúde suplementar de médio porte, localizados em seis estados brasileiros. Resultados: Foi avaliada a Curva ABC, que descreve o consumo dos produtos com maior valor utilizados pelo hospital, e demonstrado que, nas unidades hospitalares que possuem os 30 produtos mais utilizados, a maioria vem de um único fornecedor, o que, em um momento de urgência, dificulta a agilidade na reposição. Conclusão: Cada gestor deve direcionar o estoque da sua unidade hospitalar de acordo com a classificação que mais atender ao perfil da sua instituição para garantir a logística no gerenciamento dos estoques.
\end{abstract}

Palavras-chave: Gestão hospitalar; Logística; Suprimentos; Rede privada; Médio porte.

\begin{abstract}
Background: The rational management of stocks consists of an indispensable tool for a hospital unit to manage in a competitive manner, especially in the case of private networks that are subject to market pressure and of health insurers. Goals: Analyze the consumption of hospital supplies based on classifications that assist strategic processes in hospital management. The study is justified by the lack of parameterization and data input related to the supply items of hospitals managed by a hospital health system in Brazil in terms of centralized information for decision-making by hospital managers. Methods: The study is of an exploratory observational nature and sought the necessary subsidies to improve the relationship between Basic Indicators vs. Hospital in the management of hospital supplies. The perception of health managers and how these researched data can be applied in a structured way in managerial decisions was prioritized. The sample focused on six medium-sized general private supplementary health hospitals, located in six Brazilian states. Results: The ABC Curve that describes the consumption of the products with the highest value used by the hospital, and demonstrated that, in hospital units that have the 30 most used products, most come from a single supplier, which, in an time of urgency, hinders the agility in the replacement. Conclusion: Each manager must direct the stock of their hospital unit according to the classification that best meets the profile of their institution to ensure logistics in stock management. Keywords: Hospital management; Logistics; Supplies; Private network; Medium size.
\end{abstract}

\section{Resumen}

Introducción: La gestión racional de stocks es una herramienta indispensable para que una unidad hospitalaria se gestione 
de forma competitiva, especialmente en el caso de redes privadas que están sometidas a presiones del mercado y aseguradoras sanitarias. Objetivos: Analizar el consumo de insumos hospitalarios a partir de clasificaciones que apoyen los procesos estratégicos en la gestión hospitalaria. El estudio se justifica por la falta de parametrización y entrada de datos relacionados con los elementos de suministro de los hospitales administrados por un sistema de salud hospitalario en Brasil en términos de información centralizada para la toma de decisiones por parte de los administradores de los hospitales. Método: El estudio es de carácter exploratorio observacional y buscó los subsidios necesarios para mejorar la relación entre Indicadores Básicos x Hospital en la gestión de insumos hospitalarios. Se priorizó la percepción de los gerentes de salud y cómo estos datos investigados se pueden aplicar de manera estructurada en las decisiones gerenciales. La muestra se centró en seis hospitales de salud complementarios privados generales de tamaño medio ubicados en seis estados brasileños. Resultados: Se evaluó la Curva ABC, que describe el consumo de los productos de mayor valor utilizados por el hospital, y se demostró que, en las unidades hospitalarias que cuentan con los 30 productos más utilizados, la mayoría proviene de un solo proveedor, el cual, en un momento, la urgencia, entorpece la agilidad en la reposición. Conclusión: Cada gerente debe dirigir el stock de su unidad hospitalaria según la clasificación que mejor se adapte al perfil de su institución para asegurar la logística en la gestión de stock.

Palabras clave: Gestión hospitalaria; Logística; Suministros; Red privada; Tamaño medio.

\section{Introdução}

A importância do campo da logística vem crescendo cada vez mais em todos os segmentos organizacionais que prestam serviços e necessitam da aquisição e da manutenção de insumos específicos e de uso geral. Esse processo não foi diferente no caso dos hospitais, já que a variedade imensa de itens utilizados nos atendimentos, associada ao risco da falta de um insumo, pode comprometer a saúde dos pacientes, além concentrar estoques com excesso de produtos, imobilizando recursos financeiros em espaços mal organizados, que poderiam ser aplicados em outras áreas da instituição.

Por isso mesmo, a gestão racional dos estoques já se tornou uma ferramenta indispensável para que uma unidade hospitalar possa ser gerida de maneira competitiva, principalmente no caso das redes privadas que fazem parte do escopo deste trabalho de pesquisa e estão sujeitas à pressão do mercado e também das seguradoras de saúde. Como a administração da logística hospitalar precisa atender ao mesmo tempo a todos os trâmites financeiros e operacionais, isso requer um posicionamento estratégico da instituição, capaz de dar conta de toda a cadeia de suprimentos.

Cabe lembrar que as informações coletadas nas diversas áreas são reflexos de dados recolhidos que se relacionam entre si para elucidar o contexto geral de uma instituição. Quando analisados, eles se transformam em informações relevantes para orientar na direção a ser tomada em situações simples e complexas que visam a melhoria na assistência prestada em diversos nichos, desde o controle rigoroso dos recursos materiais e/ou profissionais envolvidos até a gestão mais adequada em termos de custos na prestação de assistência médica de qualidade, principalmente nos hospitais particulares.

Como são muitas as adversidades da rotina de um hospital, tanto nos aspectos operacionais quanto estratégicos e gerenciais, Mezomo (1995) é categórico ao afirmar que o processo de gestão em saúde exige medidas de planejamento, alocação de recursos escassos, avaliação de desempenho e outras atividades básicas de administração, além de reuniões de equipe, padronização de procedimentos, coordenação, direção e controle. Na visão de Vecina Neto e Malik (2007), os sistemas informatizados auxiliam na competitividade empresarial e também contribuem para a melhoria do atendimento à comunidade em um serviço de necessidade básica. Para os autores, é preciso ainda que sistemas seguros sejam implementados na área da saúde para evitar consequências graves, como processos judiciais ou indução ao erro médico. Portanto, é fundamental que um sistema informatizado forneça informações precisas, completas, úteis e em tempo real, mas cabe aos gestores o desafio de selecionar os indicadores que podem mostrar um panorama da instituição onde atuam.

O foco deste estudo consiste em demonstrar os indicadores na cadeia de suprimentos que são capazes de contribuir para o conhecimento da situação atual, identificando processos críticos e demonstrando possíveis sugestões para a área do estoque da instituição hospitalar. A explanação de como os indicadores influenciam a gestão da área de suprimentos começa com a visibilidade dos atendimentos aos pacientes internos e de urgência. Outro aspecto que merece destaque são os dados levantados por Galvão 
(2002), que indicam que a auditoria exerce uma função de extrema importância na redução de desperdícios de medicamentos e materiais que, muitas vezes, são ocasionados pela falta de registro do item utilizado durante o atendimento. Dessa forma, é possível corroborar no sentido de que o preenchimento correto de prontuários e fichas hospitalares consiste em um instrumento de extrema importância, à medida que traz benefícios em termos de qualidade de atendimento, minimizando os desperdícios.

No caso dos indicadores de suprimento do estoque hospitalar, o principal setor responsável pelo abastecimento de produtos estocáveis na instituição compromete grande parte do dinheiro 'parado' no hospital. Nesse sentido, a gestão das compras realizadas para o setor deve contemplar a quantidade de saída de um determinado produto pelo período que o fornecedor possa reabastecê-lo, impedindo a geração de itens sem ou com baixo consumo que ficam à disposição por muito tempo. Este indicador possibilita ao hospital a liberação de capital para investir em outras áreas, para que o dinheiro não fique estagnado.

Isso significa que o dimensionamento (o número de dias de consumo mantidos em estoque) dos insumos em estoque visa, sobretudo, a cobertura de estoque para cada item, reduzindo para quase $0 \%$ a falta do produto nos casos de atendimento. O cálculo deve levar em conta não apenas a sazonalidade, mas também as entregas atrasadas por parte dos fornecedores.

Atualmente, existem softwares com essas características, como o Business Intelligence (B.I.), o Big Data (baseado em valor, volume, velocidade, variedade e veracidade dos dados) e o Key Performance Indicator (KPI), que conseguem apoiar a gestão e validar toda a cadeia e seus processos de desempenho de produtividade, qualidade e capacidade estratégica, pois atendem às necessidades em Supply Chain Management, incluindo as curvas de classificação que maximizam os resultados e auxiliam no dimensionamento sem desperdiçar recursos. Caso a unidade não possua um sistema informatizado, é possível realizar a tabulação de dados, porém, de forma mais dispendiosa.

Nesse contexto, podemos destacar alguns dos indicadores básicos mais conhecidos, porém de extrema relevância: Curva ABC - Financeiro - Itens de alto, médio e baixo consumo; Classificação PQR - Cliente - Itens de alta, média e baixa popularidade; Classificação 123 - Fornecedor - Itens de fácil, média e difícil aquisição.

Após a classificação dos produtos estocáveis, é possível estabelecer a estratégia política mais adequada ao res-suprimento, tomando como base parâmetros como o estoque mínimo para a segurança no atendimento. Isso significa a manutenção do estoque disponível para utilização, a elaboração de um calendário de compra, em especial para períodos sazonais, bem como o planejamento da compra, do fornecimento e do res-suprimento. Vale destacar que a adequação de uma política nesse sentido é possível com um sistema hospitalar informatizado, pois os dados são mais facilmente levantados com o auxílio de um software.

O objetivo central deste artigo consiste em analisar o consumo de suprimentos hospitalares com base em classificações que auxiliam os processos estratégicos na gestão hospitalar. O estudo justifica-se em razão da carência de parametrização e do input de dados relacionados aos itens do suprimento de uma instituição gerida por um sistema hospitalar de saúde no Brasil em termos de informações centralizadas para as tomadas de decisão dos gestores hospitalares. Por isso, o levantamento de informações, com base em indicadores, visa colaborar para melhorar a atuação desses profissionais. Como a tecnologia vem se especializando neste nicho hospitalar, ela beneficia de maneira expressiva e com agilidade o fornecimento na base da gestão para decisões de curto, médio e longo prazo.

Além disso, os sistemas informatizados são grandes aliados no controle de processos para que o gerenciamento possa ser o mais eficiente possível, tendo em vista a recuperação e o bem-estar do paciente. Portanto, o gestor onde ele deve aplicar os recursos na validação de processos e no engajamento da equipe com treinamentos multifuncionais e/ou administrativas. De forma geral e sintética, esta pesquisa teve como finalidade contribuir para a formulação e para a interpretação mais acurada da gestão de suprimentos, possibilitando que seja obtida uma percepção real das condições das instituições hospitalares, com ênfase na rede privada de saúde suplementar de médio porte no Brasil. 


\section{Materiais e Métodos}

Trata-se de um estudo de natureza observacional exploratória (Estrela, 2018) que consistiu em buscar, no decorrer da pesquisa, os subsídios necessários para aprimorar a relação entre Indicadores básicos x Hospital na gestão de suprimentos hospitalares. Para isso, utilizamos a percepção de gestores da saúde e de que forma esses dados podem ser aplicados de maneira estruturada em decisões gerenciais do ponto de vista estratégico. A amostra do estudo é composta por seis hospitais gerais privados de saúde suplementar de médio porte, localizados no Distrito Federal (DF) e nos Estados de São Paulo (SP), Rio de Janeiro (RJ), Ceará (CE), Paraná (PR) e Rio Grande do Norte (RN).

A solicitação de dados para o estudo inicialmente era composta por 35 hospitais brasileiros da saúde suplementar. Porém, foram aprovadas as amostragens da coleta de dados em apenas seis hospitais privados de médio porte, espalhadas pelo território brasileiro. As instituições selecionadas foram divididas em dois grupos para efeito de estudo, as que são hospitais gerais, mas possuem maternidade e aquelas que são somente hospitais gerais. Com a inclusão da maternidade, temos os hospitais dos estados de SP e RN. Já no caso dos hospitais gerais, foram estudadas as unidades do DF, do RJ, do CE e do PR.

Por hospital de médio porte, tomamos como definição aqueles que dispõem entre 51 e 150 leitos, conforme a determinação do Ministério da Saúde (Brasil, 1987). Ao escolhermos esse nicho hospitalar, restringimos a amostra do universo a ser considerado da saúde suplementar, especificando as instituições mais propensas à utilização de sistemas informatizados para o controle de dados em seus processos. Cabe destacar que essa probabilidade é bem menor nos hospitais de pequeno porte. Para validar esta pesquisa, foram considerados apenas os hospitais privados da saúde suplementar de médio porte que já utilizam sistemas informatizados para algum tipo de controle de dados em seus processos, pois todos os hospitais aqui estudados apresentaram como requisito um sistema hospitalar informatizado próprio. Por outro lado, foram desconsiderados os hospitais que não dispõem de quaisquer tecnologias em seus processos de informação.

O estudo foi realizado durante o período de janeiro a dezembro de 2018. A pesquisa contemplou as fontes de dados primários, uma vez que estes foram coletados diretamente da fonte dos dados por extração para que pudessem servir de embasamento ao estudo (Pelizzon, 2004). Essa coleta foi realizada nos hospitais listados na amostragem, que possuem um sistema informatizado próprio, como no DF e nos Estados de SP, RJ, CE e RN, enquadrados na categoria de hospital privado e de médio porte. Os dados obtidos foram coletados por instrumentos de auto coleta, ou seja, por meio do envio dos instrumentos de extração de dados pelo correio eletrônico e pelo questionamento presencial direto junto ao gestor nacional. Para a delimitação do tempo, foi considerado um período de 30 dias para o recebimento do retorno do e-mail- Somente após o acordo com o gestor nacional das unidades de estudo, as informações foram extraídas.

\section{Resultados}

Este estudo buscou focar no apoio às tomadas de decisão dos gestores, de modo a manter o suprimento do estoque da unidade hospitalar, assim os temas abordados no estudo por meio de comparações e do cruzamento de dados entre o DF e os Estados de SP, RJ, CE, PR e RN. A análise foi direcionada da seguinte maneira: atendimentos X necessidade ao suprimento, tipos de produtos mais utilizados, produtos de maior utilização, classificação de produtos por gestão de demanda X médias móveis, curva $\mathrm{ABC}$, itens de alto risco e antimicrobianos. Os resultados foram divididos basicamente em dois tipos, os hospitais com Maternidade, em SP e no RN, e os hospitais sem Maternidade, no DF, no CE, no PR e no RJ. É preciso destacar que, a principal característica dos hospitais de médio porte aqui estudados, caracteriza-se pela capacidade normal ou de operação de 51 a 150 leitos (Martins e Waclawovsky, 2015), que está relacionada ao quesito atendimento de internações. Verificamos também que essas instituições não possuem restrições a especialidades. Por esta razão, os dados foram classificados somente por atendimentos relacionados à maternidade ou não, que é o divisor relevante entre os dados apurados. 
Ao avaliar os 30 produtos com maior utilização nas unidades, pudemos verificar que nos hospitais estudados que possuem maternidade, ou seja, em SP e no RN, temos os seis tipos de produtos listados abaixo, sendo que cada um deles apresenta uma quantidade no estoque:

Figura 1.

\begin{tabular}{|l|r|}
\hline RN & Qtd Produtos \\
\hline MATERIAL TECNICO & 15 \\
\hline MATERIAL DE CONSUMO GERAL & 9 \\
\hline MEDICAMENTO & 5 \\
\hline MATERIAL GRAFICO & 1 \\
\hline
\end{tabular}

\begin{tabular}{|l|r|}
\hline SP & Qtd Produtos \\
\hline MATERIAL TECNICO & 16 \\
\hline MATERIAL DE CONSUMO GERAL & 6 \\
\hline MEDICAMENTO & 4 \\
\hline MATERIAL DE EXPEDIENTE & 2 \\
\hline MATERIAL GRAFICO & 1 \\
\hline ROUPARIA & 1 \\
\hline
\end{tabular}

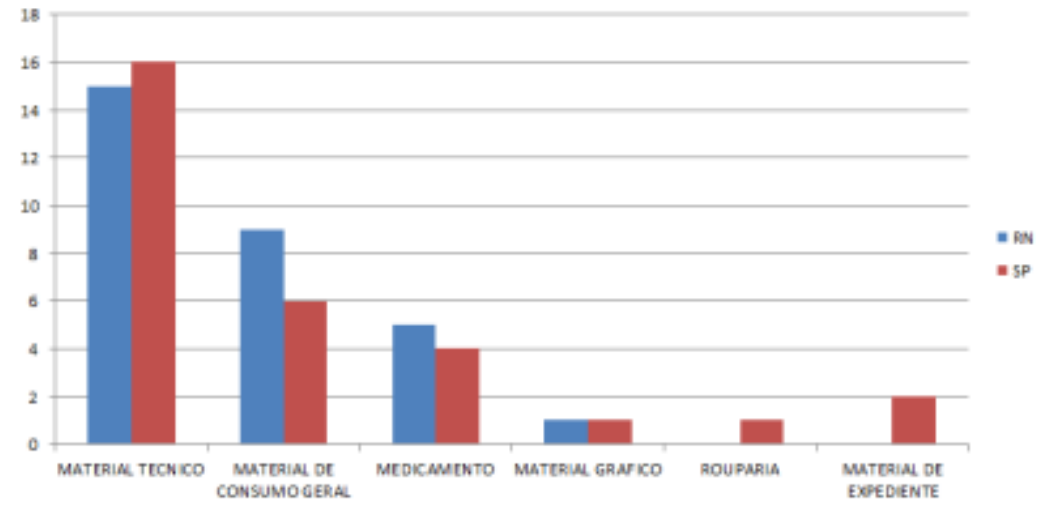

Fonte: Autores.

Observamos, ainda, que existe uma semelhança entre os tipos de produtos para esses dois hospitais, levando em consideração todos os tipos de produtos de maior utilização. São eles Material Técnico e Material de Consumo Geral, seguidos de Medicamentos. Para os hospitais avaliados que não possuem maternidade, no DF, no CE, no PR e no RJ, seguem as tabelas que registraram a quantidade de produtos relacionados a essas unidades hospitalares:

Figura 2.

\begin{tabular}{|l|r|}
\hline CE & Qtd Produtos \\
\hline MATERIAL TECNICO & 21 \\
\hline MEDICAMENTO & 4 \\
\hline MATERIAL DE CONSUMO GERAL & 2 \\
\hline MATERIAL GRAFICO & 2 \\
\hline MATERIAL DE EXPEDIENTE & 1 \\
\hline
\end{tabular}

\begin{tabular}{|l|r|}
\hline DF & Qtd Produtos \\
\hline MATERIAL TECNICO & 23 \\
\hline MEDICAMENTO & 4 \\
\hline ALIMENTO & 1 \\
\hline MATERIAL DE EXPEDIENTE & 1 \\
\hline MATERIAL GRAFICO & 1 \\
\hline
\end{tabular}

\begin{tabular}{|l|r|}
\hline PR & Qtd Produtos \\
\hline MATERIAL TECNICO & 17 \\
\hline MEDICAMENTO & 9 \\
\hline MATERIAL DE CONSUMO GERAL & 3 \\
\hline MATERIAL DE EXPEDIENTE & 1 \\
\hline
\end{tabular}

\begin{tabular}{|l|r|}
\hline RJ & Qtd Produtos \\
\hline MATERIAL DE CONSUMO GERAL & 15 \\
\hline MATERIAL TECNICO & 13 \\
\hline MATERIAL GRAFICO & 1 \\
\hline MEDICAMENTO & 1 \\
\hline
\end{tabular}

Fonte: Autores.

Foi possível notar que existe uma semelhança entre os tipos de produtos para esses quatro hospitais, levando em consideração todos os produtos de maior utilização, que são o material técnico, os medicamentos e o material de consumo geral. 
Após a análise, foi possível verificar que os itens mais utilizados por esses dois hospitais estão diretamente ligados à estrutura hospitalar, no caso dos itens que são primordiais para o atendimento e que não estão vinculados a patologias. Os itens relacionados ao tipo 'Material de Expediente', listados somente em São Paulo, foram considerados como 'Material de Consumo Geral', não distorcendo o gráfico, e sim nivelando ainda mais os dois hospitais estudados, mesmo em regiões tão distintas. Nesse aspecto, os itens de maior consumo são os materiais de limpeza e os conhecidos Equipamentos de Proteção Individual (EPIs).

Dando continuação à análise dos 30 produtos com maior utilização, foi possível constatar que, nos hospitais que não possuem maternidade (DF, CE, PR e RJ), existe um dado relevante quando os itens são separados pelas maiores quantidades utilizadas. Após essa análise, pudemos perceber que os itens mais utilizados por esses quatro hospitais estão também diretamente ligados à estrutura hospitalar, no caso dos itens que são primordiais para o atendimento. Dessa forma, foi possível visualizar melhor os itens essenciais no caso de atendimento. Os itens relacionados ao tipo Material de Expediente, Material de Consumo Geral e Material Gráfico, listados em todos os estados, podem ser considerados como ‘Material Técnico’, não distorcendo o gráfico, e sim nivelando todos os hospitais estudados. Já os itens de maior consumo são os conhecidos EPIs e os materiais de limpeza.

Como a demanda de um produto pode ser calculada por métodos variados, utilizamos, para efeito de estudo, a análise do tipo Previsões Baseadas em Médias Móveis. Esse método analítico simples pode ser facilmente implementado quando há a necessidade de uma grande quantidade de dados históricos, como é o caso aqui apresentado. Na análise dos 30 produtos com maior utilização, pudemos verificar que, nos hospitais que possuem maternidade (SP e RN), tivemos uma média muito relevante, graças a uma visão clara e, ao mesmo tempo, simplificada. Para isso, usamos o cálculo da soma de utilização dos produtos durante o ano de 2018 e dividimos esse valor por 12, total de meses, chegando ao consumo médio. Após essa análise, percebemos que os itens mais utilizados por esses dois hospitais estão diretamente ligados à estrutura hospitalar, no caso dos itens que são necessários à situação de atendimento. Com este dado simples, a unidade se mantém com itens sem grande falta, pois consegue equilibrar o estoque para que o atendimento possa receber o mínimo necessário.

Dando continuidade à análise dos 30 produtos de maior utilização, verificamos que, nos hospitais que não possuem maternidade (DF, CE, PR e RJ), obtivemos também uma média muito relevante, o que possibilitou uma visão simples e clara do uso dos insumos. Essa visão estratégica do controle de estoque, a análise mostrou que os itens mais utilizados por esses quatro hospitais, assim como os aqueles que dispõem de maternidade, estão ligados à estrutura hospitalar. Com este cálculo simples, a unidade conseguiu manter os itens sem grande falta por causa da visibilidade do estoque para que o atendimento pudesse receber o mínimo necessário.

Com relação à popularidade dos produtos, dentre os 30 itens mais utilizados, selecionamos para a classificação apenas os medicamentos, devido à solicitação ser realizada diretamente, o que não ocorre no caso dos materiais. Dessa forma, pudemos verificar que nos hospitais estudados que possuem maternidade, ou seja, em SP e no RN, encontramos os produtos abaixo, destacando que cada um possui um grupo de fármaco a ele. Apesar disso, a demonstração comparativa entre os dois hospitais estudados (RN e SP), não foi possível, devido ao cadastro da unidade hospitalar do RN não dispor de todos os produtos de maior popularidade classificados em sua base de dados.

Pudemos, então, observar apenas que existe uma semelhança na utilização dos itens, o que já era esperado no caso de hospitais semelhantes. Com relação à análise de popularidade dos 30 produtos nos hospitais que não possuem maternidade (DF, CE, PR e RJ), seguem as tabelas para mostrar que cada produto deve possuir um grupo de fármaco a ele relacionado. A análise dos dados demostrou com clareza que as quatro unidades utilizaram uma quantidade maior de águas e sais, considerado como o diluente da maioria dos medicamentos, principalmente dos endovenosos.

Avaliamos também a Curva ABC, relacionada aos produtos com maior valor. Selecionamos para essa classificação todos os tipos de produtos utilizados pelo hospital, pois, mesmo que não seja uma solicitação direta, como no caso dos medicamentos, todos os produtos contemplaram a curva $\mathrm{ABC}$ já pré-definida pelos hospitais que compõem o escopo desta pesquisa. Em relação a 
essas informações, pudemos verificar que a unidade hospitalar do RN não possui dados palpáveis para uma melhor apreciação dos seus itens, principalmente no que se refere à discriminação de produtos que exigem um cuidado maior. Já a unidade hospitalar de SP possui dados mais claros, porém ainda não totalmente direcionáveis a uma análise mais criteriosa em relação a possíveis e/ou necessários cuidados.

Quanto à análise de produtos de maior valor em hospitais que não possuem maternidade (DF, CE, PR, RJ), apenas as análises de dois deles foram realizadas, pois, como citado anteriormente, foram utilizados apenas os dados já definidos. Nesse caso, as unidades do DF e do PR não possuem dados relacionados a essa classificação. Na análise dos 30 produtos com maior utilização, pudemos constatar que, nos hospitais estudados que possuem maternidade (SP e RN), temos um dado relevante quando nos referimos à quantidade de fornecedores de um mesmo item, visando opções para o abastecimento, como mostram as tabelas.

A análise demonstrou que, nas unidades hospitalares que possuem os 30 produtos mais utilizados, a grande maioria deles vem apenas de um único fornecedor, o que, em um momento de urgência, dificulta a agilidade na reposição viável. No caso do RJ, $\mathrm{o}$ atendimento de reposição configurou-se de forma delicada, pois a maioria dos produtos possui somente um ou dois fornecedores. Já o hospital do DF possui o melhor cenário dentre os quatro avaliados, pois a maioria dos produtos possui mais de um fornecedor, o que o torna uma unidade de atendimento à saúde menos conservadora.

No que se refere ao uso de produtos denominados de alto risco nas unidades hospitalares, foram selecionados apenas os medicamentos relacionados a essa classificação. Também foram escolhidos 10 medicamentos devido à unidade do CE, sem maternidade, não possuir um cadastro bem definido e dispor apenas de 10 produtos que estão incluídos nessa classificação. Pudemos verificar que, nos hospitais estudados que possuem maternidade, ou seja, em SP e RN, os produtos classificados como de alto risco apareceram nas quantidades relacionadas.

Foi possível também observar os 10 produtos (medicamentos) com maior utilização dentro da categoria denominada de alto risco. Na classificação dos produtos de alto risco, os mais utilizados em ambas as instituições foram muito semelhantes, como deveria ser a comparação entre dois hospitais com as mesmas características. Com relação à análise de produtos de alto risco em hospitais que não possuem maternidade (DF, CE, PR e RJ), as quantidades dos produtos encontradas são mostradas a seguir: Com isso, pudemos observar que, na classificação dos produtos de alto risco, os mais utilizados em ambas as instituições não são muito semelhantes, o que veio de encontro ao esperado na comparação de hospitais com as mesmas características.

Com relação ao uso de produtos denominados antimicrobianos nas unidades hospitalares, selecionamos apenas os medicamentos, devido a esse tipo de classificação. Foram escolhidos 12 medicamentos, e não 30, devido à classificação da base de origem de SP não possuir um cadastro bem definido, contendo apenas essa quantidade de produtos classificados. Pudemos perceber que, nos hospitais estudados que possuem maternidade, ou seja, em SP e no RN, os produtos estão classificados como antimicrobianos nas quantidades descritas abaixo: Também foram elencados os 12 produtos (medicamentos) mais utilizados da categoria denominada antimicrobiana Observamos ainda que, mesmo com diferença quantitativa na classificação dos produtos antimicrobianos, os mais usados em ambas as instituições são muito semelhantes, como deveria ser na comparação de hospitais com características semelhantes.

\section{Discussão}

Para efeito desta pesquisa, analisamos algumas classificações e a curva para o res-suprimento, demonstrando onde cada classificação poderia auxiliar na compreensão da utilização, fornecendo o apoio necessário ao gestor para visualizar melhor a instituição e, com base em dados reais, estabelecesse o cruzamento das informações. Como já foi elucidado anteriormente, este estudo contemplou as seguintes classificações: Curva ABC, Classificação PQR, Classificação 123, Classificação de Médias Móveis, Classificação itens de alto risco, Classificação de itens antimicrobianos e Classificação de tipos de produtos. 
Segundo Gianesi e Biazzi (2011), o estoque é um conjunto de bens armazenados, com características próprias, que atendem aos objetivos e às necessidades da empresa. Dessa forma, todo item armazenado em um depósito, almoxarifado, prateleira, gaveta ou armário para ser utilizado pela empresa em suas atividades - de produção ou administrativa - é considerado um item do estoque da organização. A administração eficiente dos estoques cria uma vantagem competitiva com os demais concorrentes, pois é capaz de diminuir os custos, reduzir o tempo de atendimento e, consequentemente, melhorar a qualidade e o atendimento oferecido pelo próprio hospital.

Em relação à falta de materiais, podem ser abordadas três causas principais dentre as mais indicadas em materiais de logística e supply-chain. As causas estruturais estão relacionadas à falta de investimentos por parte no setor pela burocracia nos processos e a centralização das causas organizacionais que englobam os corriqueiros problemas de determinação insuficiente para atingir os objetivos da equipe, a falta de profissionalismo dos gestores, a pouca capacitação e a atualização de pessoal, além de recursos financeiros escassos e a ausência de planejamentos em longo prazo. Já as causas individuais acabam por derivar das anteriores ao mostrar diretores mal preparados e funcionários desmotivados para completar esse panorama.

Nos casos em que há um tempo maior de análise, a previsão é usada para determinar quando um evento poderá ocorrer, para, então, obtermos os dados para as decisões a serem tomadas. Portanto, a previsão é um fator efetivo e eficiente como ferramenta de planejamento, mas precisamos levar em consideração dois tipos de eventos, os externos incontroláveis, como a economia, o governos, os clientes e os competidores e os internos controláveis, como o atendimento e as informações de estatísticas de ocupação. Fazer a previsão desses eventos se aplica somente aos fatores internos controláveis, enquanto as tomadas de decisão se aplicam a ambos.

O vínculo com a previsão de demanda e a liderança aumenta à medida que se deseja mitigar as chances e os riscos, por meio de um olhar mais científico para lidar com o ambiente. Segundo Makridakis, Wheelwright e Hyndman (1998), as áreas em que a previsão possui um papel preponderante são: Planejamento (uso eficiente dos recursos requer um planejamento de transportes e recursos financeiros); Aquisição de recursos (esse planejamento é necessário para determinar as futuras solicitações de recursos em diversas áreas); Determinação de requerimento de recursos (as organizações devem determinar os recursos a serem adquiridos em longo prazo).

Ainda de acordo com os autores, os tipos de previsão podem ser divididos em curto prazo, médio prazo e longo prazo. Dependendo da previsão, a organização deve ampliar o uso de diferentes ferramentas, aumentando a capacidade de prever pela utilização de múltiplos métodos. Para lidar com as diversas situações e os diferentes ambientes, foram desenvolvidas algumas metodologias práticas. Uma delas é a quantitativa, pois os métodos quantitativos são definidos como técnicas estatísticas que analisam os comportamentos, as mudanças e as oscilações das séries temporais.

Com a responsabilidade de manter o estoque de medicamentos advindos de fornecedores, laboratórios ou de outros fabricantes, que ficarão sob a responsabilidade do hospital e serão destinados aos seus pacientes, as utilizações geradas pelo atendimento e o momento do res-suprimento dos estoques muitas vezes leva a dúvidas e a variações que podem ser geradas em períodos sazonais, ou mesmo em um período de uma epidemia sem prévio aviso. Portanto, para uma boa gestão dos estoques da farmácia, é importante que haja muita organização e atenção no armazenamento dos insumos para que não isso seja feito de maneira excessiva, o que geraria muito dinheiro 'parado' para o hospital.

Também vale destacar a necessidade de um controle capaz de impedir a falta de materiais e de medicamentos para o cliente final, ou seja, o paciente na situação de atendimento hospitalar. Nesse sentido, gerir os estoques da farmácia assume um caráter primordial, visto que é preciso atender às necessidades de materiais e de medicamentos de todo o hospital, elencando aqueles de maior e de menor demanda, buscando encontrar o equilíbrio no abastecimento da unidade para assegurar um atendimento ágil no momento em que o produto é requerido.

O termo "Gestão de Demanda" começou a ser discutido por meio da definição da palavra demanda que, segundo Proud 
(2012, p. 568), significa "uma necessidade para um produto ou componente particular". Em longo prazo, ele indica uma base para decisões estratégicas, como a ampliação das instalações, uma vez que seu intuito é prover o grau necessário de assertividade, considerando as previsões em termos de demanda. Por isso mesmo, a influência da demanda é um fator a ser pesquisado para que seja possível obter o melhor aproveitamento dos recursos existentes nos momentos em que a utilização se sobrepõe à curva da demanda e, concomitantemente, um aumento nos volumes dos estoques é considerado inadequado. Dessa forma, Proud (2012) e Pires (2004), concluem que o processo de gestão da demanda pode ser representado por macro atividades que visam predizer o consumo dos medicamentos de forma que a aquisição possa ser nas quantidades apropriadas.

Assim como as macro atividades, a gestão de demanda compreende vários tipos de demanda. É fundamental conhecê-las e realizar a análise para identificar em qual delas os medicamentos disponíveis na instituição se enquadram. A primeira delas é a Demanda Permanente, para produtos com vida longa que requerem res-suprimento periódico. Na Demanda Sazonal, a sazonalidade pode ser definida como o conjunto dos movimentos ou flutuações com período igual ou inferior a um ano, sistemáticos, mas não necessariamente regulares, que ocorrem numa série temporal, sendo resultado de causas naturais, econômicas, sociais e institucionais.

Há também a Demanda Irregular, que depende de outros fatores, como taxas de câmbio, promoção e propaganda. Já a Demanda em Desuso ocorre quando a demanda do produto acaba ou um novo produto ocupa o seu lugar, e, por fim, a Demanda Derivada que, associada a outro produto, pode aumentar ou diminuir. Existem ainda os métodos de controle da previsão da demanda, onde a maioria dos modelos de previsão está vinculada a séries temporais. Essa técnica consiste em analisar o comportamento da variável temporal e, a partir dela, projetar novos valores para o futuro. Nesse sentido, as informações básicas permitem decidir quais serão as dimensões e a distribuição no tempo da demanda de medicamentos, podendo ser classificadas em duas categorias:

a) Técnicas qualitativas: dependem exclusivamente da expertise do(s) previsor(es), sendo geralmente mais caras e trabalhosas que os métodos quantitativos de previsão. São ideais para situações que não apresentam séries históricas disponíveis e/ou o julgamento humano é necessário, sendo desenvolvidas por meio de pesquisas de opinião, painéis e reuniões de especialistas que consideram a evolução do consumo no passado, analisando as variáveis cuja evolução está ligada diretamente ao consumo e à influência do marketing farmacêutico.

b) Técnicas quantitativas: utilizam dados históricos de consumo como base para a determinação de padrões que podem se repetir no futuro, buscando relacionar o consumo (variável dependente) com outros fatores (variáveis independentes). Com isso, a demanda de um medicamento pode ser calculada pelos vários métodos, como as Previsões Baseadas em Médias Móveis, que se trata de um método fácil de ser implementado, mas possui limitações na prática, uma vez que exige uma grande quantidade de dados históricos. No caso das Previsões Baseadas nas Médias Móveis Ponderadas são atribuídos PESOS (ou fatores de importância) diferentes em cada período. A somatória dos pesos no período definido (semanal, mensal, trimestral, semestral, anual etc.) deve ser igual a $100 \%$ e poderá ser diferente de $100 \%$ quando o período for alterado.

Dentre as principais melhorias trazidas pelos softwares, estão a agilidade, a organização e a segurança dos dados, itens de extrema importância para a área da saúde, em razão do atendimento diário a milhares de pessoas que, muitas vezes, encontramse em situações delicadas, demandando rapidez e eficácia nos cuidados presados. Por esse motivo, os softwares não são aplicados apenas à área de atendimento direto, mas também às áreas de retaguarda, como o estoque do hospital. É possível comprovar que as ferramentas de tecnologia da informação facilitam a execução de pequenas até grandes tarefas, além de agregar eficiência ao supply-chain management. Com sistemas informatizados, podemos ter planos de res-suprimentos feitos com maior celeridade, compras em tempo menor, com preços e prazos melhores e a mesma qualidade. Portanto, as ferramentas tecnológicas destacamse como fortes aliadas para o sucesso da gestão das instituições hospitalares.

Podemos dividir os itens em estoque como sendo cada grupo de materiais/medicamentos com suas determinadas peculiaridades estocáveis e gerenciais. Para isso, devem ser levados em consideração o preço, o consumo e os prazos de entrega 
para o res-suprimento e o giro dos mesmos. As demandas contribuem para a alta aleatoriedade, sendo interessante que o gestor desses estoques separe os produtos em grupos que possuem características gerenciais semelhantes (Ballou, 1993). Esta classificação/separação contribui para que o responsável pelos estoques classifique cada item com a atenção individualizada para cada grupo de materiais e medicamentos, pois um tipo de controle se torna cada mais eficaz para um produto que precisa ser mais controlado, enquanto outros não têm esta necessidade (Lacerda, 2002).

A Curva ABC, também conhecida como 80-20, baseia-se no teorema do economista italiano Vilfredo Pareto, no século XIX, considerado um estudo sobre a riqueza e a renda da população, no qual ele observou que uma pequena parcela, representada por $20 \%$ das pessoas, concentrava a maior parte da riqueza, que representava $80 \%$. Com base nos princípios de Pareto, criou-se a classificação estatística de materiais, baseada nas quantidades utilizadas e no seu valor (Duarte e Ferreira, 2006). Isso significa que a classificação $\mathrm{ABC}$ permite controlar os itens estocados, utilizando o critério de investimento de cada um deles.

Vale ressaltar que o objetivo da classificação é identificar aqueles itens que necessitam/justificam maior atenção e tratamento adequado quanto à administração da unidade hospitalar. Esse método ganhou uma enorme proporção ao se tornar uma ferramenta gerencial bastante simples e extremamente eficaz na classificação dos itens de materiais e de medicamentos que compõem o estoque, principalmente quanto à sua importância financeira. Segundo este procedimento, os materiais/medicamentos de consumo podem ser divididos em três classes distintas (Reis e Perini, 2008):

- Classe A: abriga o grupo de itens mais importantes que devem receber uma atenção especial da administração, correspondendo a um pequeno número de medicamentos. Cerca de $20 \%$ dos itens representam $80 \%$ do valor total do estoque e são responsáveis pelo maior faturamento organizacional.

- Classe B: representa um grupo de itens em situação intermediária entre as classes A e C. Seu controle pode ser menos rigoroso que os itens de classe $\mathrm{A}$, porque representam um valor intermediário no faturamento das empresas.

- Classe C: engloba itens menos importantes que justificam a pouca atenção por parte da administração. Ela agrupa cerca de $70 \%$ dos itens, cuja importância em valor é pequena, representando cerca de $20 \%$ do valor total do estoque e pouca importância no faturamento das instituições.

Figura 3.

\section{Curva de Pareto ou curva ABC ou curva 80-20}

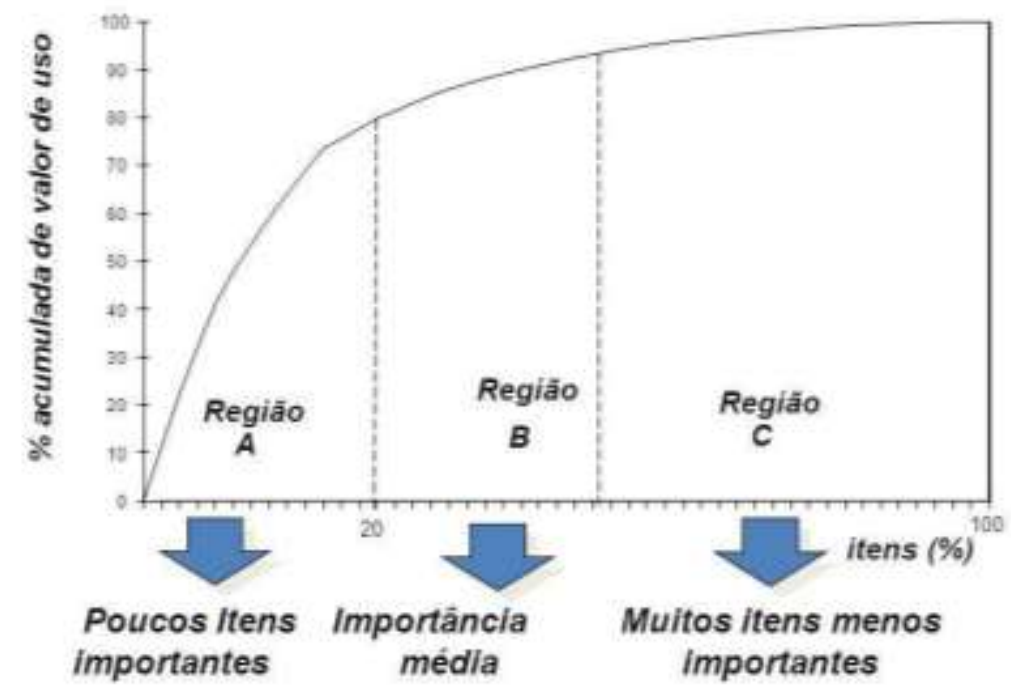

Fonte: Autores. 
Na gestão de estoques, por exemplo, costumam ser utilizadas as curvas decrescentes de Valor de Estoque e Valor dos Itens com Baixa Movimentação que também possuem uma utilização para ponto de res-suprimento com o Valor de Consumo. Com estas duas curvas, podemos estabelecer parâmetros de gestão diferenciados, fugindo à relação 80/20, relacionada à Classificação ABC. Ainda cabe acrescentar que o mais importante é o conceito da distribuição da Curva, pois a relação "80/20" não é imperativa.

O cadastro ideal requer uma revisão permanente para atualizar as informações devido à diversidade de laboratórios, fabricantes e distribuidores que existem no mercado. Essas informações se transformam em estratégicas e asseguram uma confiabilidade maior nas negociações, alertando quanto a possíveis impedimentos regulatórios para o processo de aquisição. Por isso, as informações fundamentais da empresa devem estar bem descritas, sistematizadas e acessíveis, sendo importante citar o nome do fornecedor (jurídico e fantasia), o endereço completo (telefones, cidade, CEP), o CNPJ, a nacionalidade da empresa, a Instituição financeira (bancos em que possuem conta), o endereço eletrônico (setor de compras, licitação e dos representantes) e a lista de itens/produtos com os quais essas organizações trabalham.

Com o levantamento completo dos dados sobre o fornecedor, a Classificação 123 também é conhecida como Classificação de Aquisição (Horr, Cipriano e Rabello, 1989), pois possui a função de conceder as informações necessárias ao processo de aquisição a respeito da dificuldade em adquirir um determinado item na unidade hospitalar. Sua aplicação conta com o julgamento dos profissionais do setor de compras que desempenham atividades de reabastecimento na organização, estando sujeitos à subjetividade.

Os critérios que determinam cada categoria são os seguintes: Classe 1 - Complexa; Classe 2 - Difícil; e Classe 3 - Fácil. Os itens considerados como de aquisição complexa são muito difíceis de serem obtidos, pois apresentam fatores complicadores ao serem adquiridos pelos hospitais. Dentre esses fatores, destacam-se longos setups e lead-times, ou seja, a demora do tempo de resposta, as distâncias e as variabilidades na oferta e/ou demanda, além dos riscos em relação à pontualidade, à sazonalidade, à qualidade e às fontes alternativas (cartéis). Ainda no que se refere aos itens de aquisição difícil, os fatores complicadores podem estar presentes, mas apenas aqueles que foram mencionados. Com isso, o processo de obtenção se torna relativamente difícil, já os de fácil aquisição abrangem os itens de fornecimento rápido e pontual, independentemente dos fatores complicadores.

Na aplicação das regras de classificação de produtos para a saúde, segundo a Anvisa, deve ser considerada a finalidade indicada pelo fabricante que determina a regra e a classe de risco do produto e não a classe de risco atribuída a outros produtos similares. É o uso indicado e não o uso acidental do produto que determina o seu enquadramento sanitário. Caso um produto médico realize funções que possam ser enquadradas em classes de risco diferentes, deve-se adotar a classe de risco mais crítica. Cabe ressaltar que as partes e os acessórios dos materiais, quando registrados ou cadastrados separadamente, enquadram-se de forma independente, considerando as suas características e as suas finalidades de uso, exceto no caso de serem partes e acessórios de equipamentos médicos ativos implementáveis.

Os antimicrobianos podem ser classificados de várias maneiras, "considerando seu espectro de ação, o tipo de atividade antimicrobiana, o grupo químico ao qual pertencem e o mecanismo de ação" (Viana, 2010, p.33). O sucesso no tratamento antimicrobiano depende de três importantes elementos: o fármaco, o hospedeiro e o micro-organismo. Essas relações devem ser cuidadosamente estudadas para que os resultados do tratamento escolhido não sejam comprometidos.

Os produtos utilizados no hospital possuem cada um uma classificação no Brasil, regida pela Anvisa. Dentre eles, podemos destacar os Materiais Técnico Hospitalar ou Materiais Médico Hospitalar. Eles podem ser entendidos como itens básicos para o atendimento aos pacientes: aventais descartáveis, toucas, luvas descartáveis, seringas, ataduras, campo operatório e muitos outros. São considerados como produtos hospitalares todos os aparelhos, acessórios ou materiais que estão acoplados à proteção e defesa da saúde coletiva ou individual. Os produtos para saúde são classificados em quatro classes de risco, com base nas regras definidas na RDC 185/01, que estão diretamente associadas às características específicas de sua utilização: Classe I - baixo risco; Classe II - médio risco; Classe III - alto risco; Classe IV - máximo risco. 
Já os Materiais Consumo Hospitalar são usados no dia a dia dos atendimentos médicos. Como não podem ser reutilizados, precisam ter os estoques reabastecidos periodicamente. São materiais cuja duração é limitada a curto espaço de tempo. Exemplos: artigos de limpeza e higiene, material elétrico e de iluminação, de escritório, gêneros alimentícios, artigos de mesa e combustíveis, dentre outros. Eles devem estar presentes em consultórios médicos, clínicas, postos de atendimento e em ambientes onde exista o risco de contaminação por material biológico, fungos, micro-organismos e outros agentes nocivos (Pinto, 2002).

Na categoria Medicamentos, são considerados os produtos farmacêuticos elaborados com finalidade profilática, curativa, paliativa ou para fins de diagnóstico. O papel fundamental desses produtos é prevenir doenças, recuperar a saúde e aliviar sintomas. São itens usados na rotina de atendimentos médicos que não podem ser reutilizados, necessitando que os estoques sejam reabastecidos periodicamente (Pignarre, 1999). Dentro desse tipo de produto, temos várias classificações, uma delas é o Grupo ao qual eles pertencem. O grupo relacionado ao medicamento indica onde será a principal atuação da substância, ou seja, no sistema circulatório, hematopoiético, respiratório, digestivo, geniturinário, antialérgicos ou na nutrição do paciente que o recebe (Chetley, 1995).

\section{Conclusão}

Com base na pesquisa realizada na coleta de dados, podemos afirmar que é imprescindível que o estoque hospitalar tenha sempre um acompanhamento de perto e permanente, desde a chegada até ao armazenamento dos produtos, colocando-se à frente da demanda juntamente com um processo de reposição periódica ou sazonal. Para que a análise de dados possa ser mais rápida e objetiva, a implementação de um software que se ajuste às necessidades dos pacientes é de fundamental importância, facilitando assim a análise de classificações dos produtos e a agilidade do ponto de vista do fornecimento e da reposição dos insumos necessários.

Quanto aos tipos de produtos utilizados nas unidades hospitalares estudadas, podemos considerar que todos os hospitais estudados se assemelham entre os tipos de produtos, em especial no caso do Material Técnico e do Material de Consumo Geral, seguidos do Medicamento, independentemente da unidade, possuir ou não, maternidade em sua estrutura. Dessa forma, podemos concluir que os produtos de Material de Consumo Geral e Material Técnico são os mais utilizados e que os itens de maior consumo são os EPIs e os materiais de limpeza.

A classificação de produtos por Gestão de Demanda em relação a Médias Móveis incluiu todos os produtos usados nas unidades de atendimento e, de forma simples e clara em termos de cálculo, conseguimos identificar os insumos mais utilizados por todos os hospitais, associados também à estrutura hospitalar. Com base nesse cálculo simples, as unidades conseguem manter o seu estoque, pois possuem uma boa visibilidade para que o atendimento receba o mínimo de suprimentos necessários. Foi possível concluir ainda que cada gestor pode direcionar o estoque da unidade hospitalar onde trabalha, seguindo a classificação que mais atender ao perfil da sua instituição para garantir a viabilidade do gerenciamento.

Com a finalidade de deixar cada vez mais claro para o gestor os dados que estão sendo geridos, a Curva ABC seria, inicialmente, o item que merece maior atenção, em razão ao já conhecido e exposto inicialmente '80-20', ou seja, a classificação A, que considera itens de altíssima prioridade, na qual $80 \%$ do valor de todo o estoque se concentra em apenas $20 \%$ dos itens estocados. Outro ponto a ser mencionado para melhorar a gestão dos estoques, seria a realização periódica de inventários, baseados também na Curva $\mathrm{ABC}$, levando sempre em consideração à exatidão do estoque físico e daquele representado no software de gestão.

Como sugestão para futuras pesquisas acadêmicas focadas nesse tema, indicamos três áreas da instituição hospitalar aqui elencadas inicialmente como macro áreas, para que seja possível trabalhar com uma visão completa e estrutural das atividades desenvolvidas em um hospital, bem como todos os seus indicadores, com base nos números relacionados à ocupação, ao centro cirúrgico e ao faturamento das unidades de atendimento à saúde da rede privada. 
Research, Society and Development, v. 10, n. 13, e91101321076, 2021

(CC BY 4.0) | ISSN 2525-3409 | DOI: http://dx.doi.org/10.33448/rsd-v10i13.21076

\section{Agradecimentos}

Este estudo foi financiado pela CAPES. É produto parcial de pesquisa de Mestrado desenvolvida na Escola Paulista de Medicina, da Universidade Federal de São Paulo, e aprovada pelo Comitê de Ética em Pesquisa da Universidade.

\section{Referências}

Ballou, R. H. (1993). Logística empresarial: transportes, administração de materiais e distribuição física. Atlas.

Brasil. (1987). Ministério da Saúde. Secretaria Nacional de Organização e Desenvolvimento de Serviços de Saúde. Terminologia Básica em Saúde. Disponível em: <http://bvsms.saude.gov.br/bvs/publicacoes/0112terminologia1.pdf>. Acesso em: 06 de setembro de 2020.

Chetley, A. (1995). Medicamentos problema. In Medicamentos problema (pp. 419-419).

Duarte, I. G., \& Ferreira, D. P. (2006). Uso de indicadores na gestão de um centro cirúrgico. Rev. adm. saúde, 63-70.

Estrela, C. (2018). Metodologia científica: ciência, ensino, pesquisa. Artes Médicas.

Galvão, C. R. (2002). Estudo do papel da auditoria de enfermagem para a redução dos desperdícios em materiais e medicamentos. Mundo saúde (Impr.), $275-282$.

Gianesi, I. G. N., \& de Biazzi, J. L. (2011). Gestão estratégica dos estoques. Revista de Administração, 46(3), 290-304.

Horr, L., Cipriano, Z. M., \& Rabello, E. S. (1989). Proposta de controle de material de consumo. Revista Gaúcha de Enfermagem, $10(1), 24$.

Lacerda, L. (2002). Logística reversa: uma visão sobre os conceitos básicos e as práticas operacionais. Rio de Janeiro: COPPEAD/UFRJ, 6.

Martins, C. C., \& Waclawovsky, A. J. (2015). Problemas e desafios enfrentados pelos gestores públicos no processo de gestão em saúde. Revista de Gestão em Sistemas de Saúde, 4(1), 100-109.

Mezomo, J. C. (1995). Gestäo da qualidade na saúde: princípios básicos. In Gestäo da qualidade na saúde: princípios básicos (pp. 301-301).

Pellizzon, R. D. F. (2004). Pesquisa na área da saúde: 1. Base de dados DeCS (Descritores em Ciências da Saúde). Acta Cirúrgica Brasileira, 19 , $153-163$.

Pignarre, P. (1999). que é o medicamento?, O. Editora 34.

Pinto, C. V. (2002). Organização e gestão da manutenção.

Pires, S. R. (2004). Gestão da Cadeia de Suprimentos (Supply Chain Management). Conceitos. Estratégias, Práticas e Casos. S. Paulo: Atlas.

Proud, J. F. (2012). Master scheduling: A practical guide to competitive manufacturing (Vol. 16). John Wiley \& Sons.

Reis, A. M. M., \& Perini, E. (2008). Desabastecimento de medicamentos: determinantes, consequiências e gerenciamento. Ciência \& Saúde Coletiva, 13, 603-610.

Vecina Neto, G., \& Malik, A. M. (2007). Trends in hospital care. Ciência \& Saúde Coletiva, 12(4), 825-839.

Vianna, J. J. (2010). Administração de materiais: um enfoque prático. Atlas.

Wheelwright, S., Makridakis, S., \& Hyndman, R. J. (1998). Forecasting: methods and applications. John Wiley \& Sons. 